\title{
ARTIGO CIENTÍFICO \\ Antibiose de acessos de feijão-fava infestados com gorgulhos (Zabrotes subfasciatus) em três gerações
}

\section{Antibiosis of lima beans access infested with weevil (Zabrotes subfasciatus) in three generations}

\author{
José Edmir Girão Filho ${ }^{*}$, Luiz Evaldo de Moura Pádua², Jayara Dayany da Costa Silva ${ }^{3}$, Paulo Roberto Ramalho e Silva ${ }^{4}$ \\ Westerlanya Rodrigues Medeiros ${ }^{5}$
}

\begin{abstract}
Resumo: O feijão-fava, Phaseolus lunatus L., além de ser uma importante fonte proteica para populações do nordeste brasileiro, contribui também para aumento da renda familiar das mesmas. Um dos problemas da cadeia produtiva desta espécie é o ataque de insetos durante o período de armazenamento, causado principalmente pelo gorgulho Zabrotes subfasciatus. Através de teste de confinamento avaliou-se 23 acessos de feijão-fava infestados com Z. subfasciatus, durante três gerações do inseto, objetivando buscar fontes de resistência antibiótica. A pesquisa foi conduzida em laboratório sob condições ambientais controladas. Após confinar um casal deste inseto juntamente com dez grãos de cada acesso de feijão fava por sete dias, avaliou-se o peso seco dos insetos, número de ovos, viáveis e inviáveis, emergência de adultos e período médio de desenvolvimento. Observou-se que houve efeito da troca brusca de substrato alimentar/oviposição na postura das fêmeas, houve influencia dos acessos no peso, oviposição, mortalidade e período médio de desenvolvimento dos insetos, fatos que caracterizaram o acesso UFPI 468 altamente suscetível, o acesso UFPI 701 como o mais resistente e os acessos UFPI 121, UFPI 222, UFPI 281, UFPI 504, UFPI 579, UFPI 689 Rajada, UFPI 701 e UFPI 720 como moderadamente resistentes à Z. subfasciatus.
\end{abstract}

Palavras-chave: Phaseolus lunatus; Grãos armazenados; Resistência de plantas

\begin{abstract}
The lima beans, Phaseolus lunatus L., is an important protein source for the Brazilian northeastern people. It also contributes to the income rise of those families income. One of the problems in the productive chain of this specie is the pest attack during the storage time. This situation is mainly caused by Z. subfasciatus. Twenty three lima beans accesses infested by Z. subfasciatus were evaluated during three generations of weevil through confinement test. This procedure aimed to find resistance against this plague. The research was conducted at the Laboratory of Plant Protection (UFPI) under controlled environmental conditions. A couple of this insect was confined with 10 lima beans grains. After seven days, evaluated the dry weight of insects, number of eggs, viable and non-viable, adult emergence and mean development period. It was observed that there is an effect of the sudden change in feed/oviposition substrate posture of females, the weight, oviposition, mortality and mean development period of insects were influenced by access, facts that characterized the UFPI 468 access as highly susceptible, the UFPI 701 as more resistant and UFPI 121, 222 UFPI, UFPI 281, 504 UFPI, UFPI 579, 689 UFPI Gust, UFPI UFPI 701 and 720 as moderately resistant to Z. subfasciatus.
\end{abstract}

Key words: Phaseolus lunatus; Resistance of plants; Stored grains

\footnotetext{
*Autor para correspondência

Recebido para publicação em 11/04/2016; aprovado em 12/06/2016

${ }_{1}^{1}$ Doutorando em Agronomia,Universidade Federal do Piauí, Teresina;(86) 988527148, giraofilho@ hotmail.com

${ }^{2}$ Doutor em Entomologia, Universidade Federal do Piauí, lempadua@uol.com.br

${ }^{3}$ Doutoranda em Agronomia, Universidade Federal do Piauí, dayany608@ hotmail.com

${ }^{4}$ Doutor em Biologia animal, Universidade Federal do Piauí, pramalhoufpi@yahoo.com.br

${ }^{5}$ Mestre em Agronomia, Universidade Federal do Piauí
} 


\section{INTRODUÇÃO}

O Nordeste brasileiro é responsável por aproximadamente $88,5 \%$ do total de feijão-fava, Phaseolus lunatus L., produzido no Brasil, sendo que a maior parte desta produção é de origem da agricultura familiar (IBGE, 2012). O feijão-fava é a segunda espécie do gênero Phaseolus de maior importância econômica, ficando atrás do feijão comum ou carioca, $P$. vulgaris. No entanto, seu uso na alimentação humana ainda é bastante reduzido, provavelmente por características culturais e tradicionais, além do tempo de cocção e a forma diferenciada de preparo (LOPES et al., 2010).

O gorgulho Zabrotes subfasciatus Boheman é considerado um dos principais insetos associados aos feijões armazenados, tanto $P$. vulgaris quanto $P$. lunatus (BALDIN; PEREIRA, 2010; GIRÃO FILHO et al., 2012) e seus parâmetros biológicos variam de acordo com o hospedeiro, em resposta ao seu grau de resistência (BALDIN; PEREIRA, 2010; COSTA et al., 2013; GIRÃO FILHO et al., 2012).

Muitas pesquisas têm sido realizadas com o objetivo de buscar fontes alternativas, sustentáveis, de controle de insetos, sobretudo em grãos armazenados, seja sob forma de plantas inseticidas (FRANÇA et al., 2012; GIRÃO FILHO, et al., 2014; MIKAMI et al., 2010) ou plantas resistentes (BLAIR et al., 2010; BOTTEGA et al., 2012; GIRÃO FILHO et al., 2012).

Fontes de resistência antibiótica têm sido obtidas em plantas silvestres de $P$. vulgaris, as quais têm sido utilizadas no melhoramento do feijoeiro comum com muito sucesso, graças à identificação de uma proteína, denominada Arcelina (BALNDIN; PEREIRA, 2010; BARBOSA et al., 2000). Esta proteína proporciona uma alta antibiose contra $Z$. subfasciatus (MORAES et al., 2011; ZAUGG et al., 2013). Por outro lado, pouco se sabe em relação à interação de $Z$. subfasciatus e $P$. lunatus, onde, as poucas referências que se tem, são de
GIRÃO FILHO et al. (2012) e GIRÃO FILHO et al., (2016) nas quais foram observadas variabilidade genética entre os acessos testados e indícios de antixenose, mas todos os acessos apresentaram alta suscetibilidade ao gorgulho $Z$. subfasciatus.

Neste contexto, objetivou-se avaliar vinte e três acessos de feijão-fava em relação à resistência genética antibiótica sobre Z. subfasciatus Boh. em teste de confinamento em três gerações consecutivas em condições de laboratório.

\section{METODOLOGIA}

A pesquisa foi conduzida em laboratório sob condições controladas; temperatura, $29 \pm 2{ }^{\circ} \mathrm{C}$, UR $47 \pm 10 \%$ e fotoperíodo de $12 \mathrm{~h}$. Para a criação e multiplicação dos insetos utilizaram-se recipientes plásticos de 2 litros, com tampa perfurada, contendo $500 \mathrm{~g}$ de grãos de feijão-fava (adquiridos no Mercado Municipal de Teresina) e 300 insetos adultos com 0-48 h de emergidos, provenientes de criação estoque do próprio laboratório, mantidos em feijão-fava de variedade desconhecida. A cada 15 dias após a infestação dos recipientes, retiravam-se os insetos, através de peneiramento e, após vinte dias, as observações foram diárias, a fim de conhecer a idade dos insetos.

Foram utilizados 23 acessos de feijão-fava procedentes do Banco Ativo de Germoplasma de Feijão-Fava da Universidade Federal do Piaú - BAGF/UFPI (Tabela 1) selecionados a partir de resultados de pesquisas já realizadas com feijão-fava (GIRÃO FILHO et al., 2012). Os materiais selecionados foram acondicionados em sacos de papel e colocados em freezer por 72 horas para prevenção de uma possível infestação não desejada. Antes da montagem dos ensaios, os mesmos foram postos por 72 horas no ambiente em que os bioensaios seriam realizados, para entrarem em equilíbrio higroscópico.

Tabela 1. Relação dos acessos de Phaseolus lunatus provenientes da coleção do Banco Ativo de Germoplasma de Feijão-fava da Universidade Federal do Piauí (BAGF/UFPI).

\begin{tabular}{cccc}
\hline Cód. BAGF & Nome popular & Procedência & Cor do tegumento \\
\hline UFPI-032 & - & Várzea Grande - PI & Marrom \\
UFPI-034 & Fígado de galinha & Várzea Grande - PI & Marrom \\
UFPI-121 & - & Bom Jesus - PI & Amarela \\
UFPI-122 & Fava Branca & Demerval Lobão - PI & Branca \\
UFPI-243 & - & BGH - UFV* & Laranja com preto \\
UFPI-251 & - & BGH - UFV* & Cinza \\
UFPI-281 & - & Barra da Alcântara - PI & Creme rajada \\
UFPI-463 & Fava preta & Arraial do Piauí - PI & Preta \\
UFPI-468 & Fava miudinha & São Domingos - MA & Creme \\
UFPI-486 & - & São Pedro do Piauí - PI & Roxo com Marrom \\
UFPI-500 & - & Guaramiranga - CE & Creme com hilo bege \\
UFPI-504 & - & Colinas - MA & Branca \\
UFPI-515 & - & Colégio Agrícola Soinho - PI & Branca com hilo marrom \\
UFPI-579 & Boca de Moça & Campina Grande - PB & Bege claro \\
UFPI-581 & - & BGH - UFV & Marrom \\
UFPI-654 & Boca de moça & Barro duro - PI & Branca \\
UFPI-666 & Boca de moça & Palmeirais - PI & Branca \\
UFPI-676 & - & Palmeirais - PI & Branca \\
UFPI-689 & Boca de moça & Elesbão Veloso - PI & Rajada \\
UFPI-689 & Boca de moça & Elesbão Veloso - PI & Branca \\
UFPI-701 & - & Olho d'água - PI & Branca \\
UFPI-715 & - & José de Freitas - PI & Branca \\
UFPI-720 & Boca de moça & Palmeirais - PI &
\end{tabular}


Utilizou-se o teste de confinamento, delineado ao acaso com 23 tratamentos e sete repetições, no qual o efeito antibiótico dos acessos de feijão-fava à $Z$. subfasciatus foi avaliado em três gerações consecutivas. Para isso, um casal do inseto (0-24 h de emergido) foi acondicionado em um recipiente plástico de $100 \mathrm{~mL}$, com tampa perfurada e contendo 10 grãos de feijão-fava por sete dias. Após 15 dias da infestação, procedeu-se a avaliação do número de ovos viáveis e inviáveis. Decorridos 20 dias da infestação, as observações passaram a ser diária a fim de se retirar e quantificar os insetos emergidos. Estes novos insetos foram sexados e acondicionados em tubos de ensaios distintos, machos e fêmeas, até atingir a quantidade suficiente, sete casais com 0-24 h, para uma nova infestação no mesmo acesso no qual emergiram. Feita a nova infestação, os novos insetos que ao emergir logo eram coletados, acondicionados em tubos de ensaios e levados ao freezer a $0{ }^{\circ} \mathrm{C}$ por 48 horas e posteriormente secos em estufa a $50{ }^{\circ} \mathrm{C}$ por $48 \mathrm{~h}$ para posterior pesagem (BALDIN; PEREIRA, 2010). Dessa mesma forma procedeu-se até a geração F2. Os insetos emergidos na geração F3 só foram analisados pelo fator peso.

Foram avaliados: número de ovos viáveis, número de ovos inviáveis, percentual de insetos emergidos, peso de machos, peso de fêmeas e período médio de desenvolvimento (PMD), de acordo com a fórmula;

$$
\text { PMD }=\frac{\sum \text { insetos emergidos } * \text { dia de emergência após infestação }}{\text { número de insetos emergidos }}
$$

(BARBOSA et al., 2000).

Consideraram-se viáveis os ovos de coloração branca ou leitosa, sendo os translúcidos considerados inviáveis (RIBEIRO-COSTA et al., 2007)

$\mathrm{Na}$ avaliação de emergência, as parcelas que passaram três dias consecutivos sem emergência de adultos foram descartadas

Para realização das análises de variância, os dados provenientes dos pesos e oviposição foram transformados em $\sqrt{\mathrm{x}+0,5}$ e, quando significativos ao teste $\mathrm{F}$, as médias foram comparadas pelo teste de Tukey a $5 \%$ de probabilidade.

Os dados de emergência de adultos foram transformados em arcoseno $\sqrt{\frac{\mathrm{p} \%}{100}}$ e analisados da mesma forma já descrita. $\mathrm{O}$ mesmo procedimento foi dado para as análises de PMD, que não foram transformados para tal processo, porque apresentaram os pré-requisitos da análise de variância.

\section{RESULTADOS E DISCUSSÃO}

Observou-se que houve efeito dos tratamentos em relação ao peso dos insetos, tanto de machos como de fêmeas, dentro e entre as três gerações avaliadas (Tabela 2).

Tabela 2. Médias de peso seco de machos e fêmeas de Zabrotes subfasciatus criados em acessos de feijão fava, em teste sem chance de escolha, em três gerações sob condições controladas.

\begin{tabular}{|c|c|c|c|c|c|c|}
\hline \multirow{2}{*}{ Acessos } & \multicolumn{3}{|c|}{ Machos } & \multicolumn{3}{|c|}{ Fêmeas } \\
\hline & F1 & F2 & F3 & F1 & $\mathrm{F} 2$ & F3 \\
\hline UFPI 32 & $1,03 \mathrm{ab} \mathrm{A}$ & $1,05 \mathrm{ab} \mathrm{B}$ & 0,97 abcd $\mathrm{C}$ & 1,91 abc $\mathrm{A}$ & 1,88 abc $\mathrm{A}$ & $1,84 \mathrm{ab} \mathrm{A}$ \\
\hline UFPI 34 & $1,04 \mathrm{ab} A$ & 0,97 abcde B & 0,89 bcd C & 1,78 abcde $A$ & $1,78 \mathrm{bcd} A$ & $1,75 \mathrm{ab} A$ \\
\hline UFPI 121 & $0,95 \mathrm{ab} A$ & 0,99 abcde $\mathrm{A}$ & 0,96 abcd $A$ & 1,74 abcde $A$ & $1,92 \mathrm{ab} B$ & $1,73 \mathrm{ab} A$ \\
\hline UFPI 222 & $0,96 \mathrm{ab} A$ & 0,94 bcde A & 0,96 abcd $A$ & 1,69 e $A$ & $1,65 \mathrm{~d} A$ & $1,62 \mathrm{~b} \mathrm{~A}$ \\
\hline UFPI 243 & $1,02 \mathrm{ab} A$ & 0,98 abcde $\mathrm{A}$ & 0,87 bcd B & 1,81 abcde $A$ & 1,79 abcd $A$ & 1,66 ab B \\
\hline UFPI 251 & 1,04 a A & 0,96 bcde $\mathrm{A}$ & 1,00 abcd $A$ & 1,74 abcde $A$ & $1,80 \operatorname{abcd} A$ & $1,79 \mathrm{ab} A$ \\
\hline UFPI 281 & $1,04 \mathrm{ab} A$ & 0,96 abcde $A$ & 0,99 abcd $A$ & 1,90 abcd $A$ & 1,76 bcd A & $1,75 \mathrm{ab} A$ \\
\hline UFPI 463 & 1,06 a A & $1,02 \operatorname{abcd} A$ & $0,94 \operatorname{abcd} A$ & 1,88 abcde $\mathrm{A}$ & 2,00 a B & $1,75 \mathrm{ab} C$ \\
\hline UFPI 468 & $1,00 \mathrm{ab} A$ & 1,00 abcde $A$ & 0,97 abcd $A$ & 1,77 abcde $A$ & $1,83 \operatorname{abcd} A$ & $1,67 \mathrm{ab} B$ \\
\hline UFPI 486 & $1,03 \mathrm{ab} A$ & 1,03 abc A & 1,09 a A & 1,83 abcde $A$ & 1,87 abc $\mathrm{A}$ & 1,89 a $\mathrm{A}$ \\
\hline UFPI 500 & $1,03 \mathrm{ab} A$ & 0,90 cde B & $1,01 \mathrm{abc} A$ & 1,85 abcde $\mathrm{A}$ & 1,74 bcd A & $1,62 \mathrm{~b} \mathrm{~B}$ \\
\hline UFPI 504 & $1,01 \mathrm{ab} A$ & 1,01 abcde $A$ & 0,97 abcd $A$ & 1,85 abcde $A$ & $1,85 \operatorname{abcd} A$ & $1,76 \mathrm{ab} \mathrm{A}$ \\
\hline UFPI 515 & $0,99 \mathrm{ab} A$ & 0,99 abcde $A$ & $1,03 \mathrm{ab} A$ & 1,78 abcde $A$ & 1,86 abc A & $1,75 \mathrm{ab} A$ \\
\hline UFPI 579 & $0,97 \mathrm{ab} A$ & $1,05 \mathrm{ab} A$ & 0,98 abcd $A$ & 1,89 abcde $A$ & 1,94 ab A & $1,82 \mathrm{ab} \mathrm{A}$ \\
\hline UFPI 581 & $1,01 \mathrm{ab} A$ & 0,97 abcde A & 0,95 abcd $A$ & 1,84 abcde $\mathrm{A}$ & $1,84 \operatorname{abcd} A$ & $1,70 \mathrm{ab} \mathrm{A}$ \\
\hline UFPI 654 & $1,03 \mathrm{ab} A$ & 0,95 bcde $A$ & 1,00 abcd $A$ & 1,80 abcde $A$ & 1,77 bcd A & $1,79 \mathrm{ab} \mathrm{A}$ \\
\hline UFPI 666 & $0,97 \mathrm{ab} A$ & 0,91 cde $A$ & 0,97 abcd A & 1,71 bde $\mathrm{A}$ & $1,75 \mathrm{bcd} A$ & $1,67 \mathrm{ab} A$ \\
\hline UFPI 676 & 1,09 a $\mathrm{A}$ & 1,10 a $\mathrm{A}$ & 0,96 abcd B & $1,92 \mathrm{ab} A$ & 1,87 abc $\mathrm{A}$ & $1,75 \mathrm{ab} A$ \\
\hline UFPI 689 Rajada & 1,04 a A & $1,05 \mathrm{ab} A$ & $1,03 \mathrm{ab} \mathrm{A}$ & 1,86 abcde $A$ & 1,87 abc $\mathrm{A}$ & $1,74 \mathrm{ab} B$ \\
\hline UFPI 689 Branca & 1,07 a $\mathrm{A}$ & $1,02 \operatorname{abcd} A$ & 1,02 abc $\mathrm{A}$ & 1,93 a $A$ & 1,9 abc $\mathrm{A}$ & $1,78 \mathrm{ab} B$ \\
\hline UFPI 701 & 0,92 b A & 0,90 de $\mathrm{A}$ & $0,87 \mathrm{~cd} \mathrm{~A}$ & 1,68 e $\mathrm{A}$ & $1,70 \mathrm{~cd} A$ & $1,71 \mathrm{ab} \mathrm{A}$ \\
\hline UFPI 720 & 1,04 a A & 0,88 e $\mathrm{A}$ & $0,85 \mathrm{~d} A$ & 1,90 abcd A & 1,84 abcd $A$ & $1,84 \mathrm{ab} \mathrm{A}$ \\
\hline UFPI 715 & $1,00 \mathrm{ab} A$ & 0,95 bcde $\mathrm{B}$ & 0,95 abcd B & 1,78 abcde $A$ & 1,75 bcd A & $1,70 \mathrm{ab} \mathrm{A}$ \\
\hline $\mathrm{F}$ & $2,73 * *$ & $5,12 * *$ & $3,60 * *$ & $3,21 * *$ & $4,47 * *$ & $2,68 * *$ \\
\hline $\mathrm{Cv} \%$ & 1,31 & 1,45 & 1,77 & 1,83 & 1,43 & 1,62 \\
\hline
\end{tabular}

** Médias seguidas de mesma letra minúscula na coluna não diferem estatisticamente pelo teste de Tukey $(p<0,01)$.

Médias seguidas de mesma letra maiúscula nas linhas não diferem estatisticamente pelo teste "t" de Student.

As menores médias observadas para peso de machos foram: $0,85 \mathrm{mg}$ no acesso UFPI $720 ; 0,87 \mathrm{mg}$ no acesso UFPI 701 e 0,87 mg em UFPI 243, todos na geração F3. Além disso, pode-se observar que os acessos UFPI 32, UFPI 34, UFPI 243, UFPI 676 e UFPI 715 reduziram significativamente o peso de machos da geração F1 para F3.
Embora não reduzindo estatisticamente o peso dos insetos de uma geração para outra, e apresentando uma das maiores médias de peso de macho e de fêmeas na geração F1, o acesso UFPI 720 demostrou efeito na redução do peso dos insetos nas gerações seguintes, de modo que se igualou ao acesso UFPI 701, que apresentou os insetos mais leves. 
Observou-se também que as médias de peso de fêmeas variaram de 1,62 mg na geração F3 no acesso UFPI 500 a $2,00 \mathrm{mg}$ na geração F2 no acesso UFPI 463. Neste caso, o acesso UFPI 222 destacou-se nas três gerações com as menores médias de peso de fêmeas, tendendo a reduzir ainda mais nas gerações seguintes.

$\mathrm{O}$ acesso UFPI 500 reduziu significativamente o peso de fêmeas da geração F1 à F3, de 1,85 mg para 1,62 mg, sendo a menor média observada nesta pesquisa, indicando antibiose (BALNDIN; PEREIRA, 2010; COSTA et al., 2013).

Costa et al. (2013) não observaram diferenças estatísticas no peso de fêmeas emergidas de diferentes genótipos de feijão comum, mas estas apresentaram-se mais leves no genótipo mais resistente (RAZ 49). Neste, também houve o menor consumo de massa seca dos grãos e menor massa seca consumida por inseto. $\mathrm{O}$ peso de machos foi estatisticamente diferente e apresentou-se menor neste mesmo genótipo. De acordo com Baldin e Pereira (2010) o fato do alimento fornecido às formas imaturas influenciar no peso final dos insetos adultos pode estar relacionado a fatores antinutricionais, que podem ser em decorrência da genética do acesso, o que caracterizaria uma resistência antibiótica.

Em feijão comum, Phaseolus vulgaris, encontram-se valores variando de 0,4 a 2,25 mg para machos e 0,7 a 3,60 mg para fêmeas de Z. subfasciatus (COSTA et al., 2013; RIBEIRO-COSTA et al., 2007;). Infelizmente não há muitos artigos envolvendo feijão-fava e $Z$. subfasciatus que possam ser utilizados como referência e, por este motivo algumas das referencias são com feijão comum, o que dificulta bastante a discussão sobre o assunto, haja visto que a resistência genética só pode ser comparada entre indivíduos da mesma espécie e sob as mesmas condições de teste.

Em relação à postura, pode-se observar que houve diferentes estímulos à oviposição, tanto entre os acessos quanto entre as gerações (Tabela 3).

Tabela 3. Médias da oviposição de Z. subfasciatus em feijão-fava em três gerações, em teste sem chance de escolha, sob condições controladas.

\begin{tabular}{|c|c|c|c|c|c|c|}
\hline \multirow{3}{*}{ Acessos } & \multicolumn{6}{|c|}{ Médias de número de ovos por fêmea } \\
\hline & \multicolumn{2}{|c|}{ Geração Parental* } & \multicolumn{2}{|c|}{ Geração F1 } & \multicolumn{2}{|c|}{ Geração F2 } \\
\hline & Viáveis & Inviáveis & Viáveis & Inviáveis & Viáveis & Inviáveis \\
\hline UFPI 32 & 14,71 & 3,29 & 41,9 a $\mathrm{A}$ & 5,4 a $\mathrm{A}$ & $42,71 \mathrm{ab} A$ & $3,57 \mathrm{ab} A$ \\
\hline UFPI 34 & 10,29 & 4,14 & 42,6 a $\mathrm{A}$ & 3,4 a $\mathrm{A}$ & $46,58 \mathrm{ab} A$ & $2,57 \mathrm{ab} A$ \\
\hline UFPI 121 & 16,71 & 4,86 & 47,7 a $\mathrm{A}$ & 3,0 a $\mathrm{A}$ & 38 ab A & $7,14 \mathrm{ab} A$ \\
\hline UFPI 222 & 17,43 & 2,29 & 39,7 a $\mathrm{A}$ & 3,1 a $A$ & $39 \mathrm{ab} A$ & $6,71 \mathrm{ab} A$ \\
\hline UFPI 243 & 15,86 & 3,29 & 42,0 a $\mathrm{A}$ & 4,0 a $\mathrm{A}$ & $42,28 \mathrm{ab} A$ & $3,14 \mathrm{ab} A$ \\
\hline UFPI 251 & 20,57 & 3,57 & 39,1 a $\mathrm{A}$ & 3,1 a $\mathrm{A}$ & 36 ab A & $1,86 \mathrm{~b} \mathrm{~A}$ \\
\hline UFPI 281 & 17,57 & 5,57 & 41,1 a $A$ & 5,7 a $\mathrm{A}$ & $35,57 \mathrm{ab} A$ & $2,28 \mathrm{ab} A$ \\
\hline UFPI 463 & 6,57 & 5 & 45,7 a $A$ & 4,4 a $\mathrm{A}$ & $36,86 \mathrm{ab} A$ & $4,14 \mathrm{ab} A$ \\
\hline UFPI 468 & 27,67 & 4 & 44,3 a $\mathrm{A}$ & 2,1 a $\mathrm{A}$ & 51,8 a A & $1,0 \mathrm{~b} \mathrm{~A}$ \\
\hline UFPI 486 & 11 & 2,57 & 42,9 a $\mathrm{A}$ & 6,9 a $A$ & $43,57 \mathrm{ab} A$ & $6,14 \mathrm{ab} A$ \\
\hline UFPI 500 & 17,71 & 2,86 & 44,3 a $\mathrm{A}$ & 2,5 a A & $40,42 \mathrm{ab} A$ & 5,0 ab B \\
\hline UFPI 504 & 20,43 & 4 & 39,9 a $\mathrm{A}$ & 3,7 a $\mathrm{A}$ & $43,85 \mathrm{ab} A$ & $3,43 \mathrm{ab} A$ \\
\hline UFPI 515 & 28 & 4 & 40,1 a $\mathrm{A}$ & 9,0 a $\mathrm{A}$ & $41,0 \mathrm{ab} \mathrm{A}$ & $5,57 \mathrm{ab} A$ \\
\hline UFPI 579 & 13,2 & 10,8 & 44,0 a $\mathrm{A}$ & 7,4 a $\mathrm{A}$ & $36,86 \mathrm{ab} A$ & 7,86 a $\mathrm{A}$ \\
\hline UFPI 581 & 15,86 & 1,86 & 38,7 a $\mathrm{A}$ & 7,7 a $\mathrm{A}$ & $39,14 \mathrm{ab} \mathrm{A}$ & $6,57 \mathrm{ab} A$ \\
\hline UFPI 654 & 16,71 & 3,57 & 43,8 a $\mathrm{A}$ & 2,3 a $A$ & $42,14 \mathrm{ab} A$ & $3,71 \mathrm{ab} A$ \\
\hline UFPI 666 & 20,5 & 4,83 & 40,7 a A & 4,1 a $\mathrm{A}$ & $43,71 \mathrm{ab} A$ & $2,57 \mathrm{ab} A$ \\
\hline UFPI 676 & 10,71 & 5,57 & 46,9 a $\mathrm{A}$ & 2,7 a $A$ & $44,43 \mathrm{ab} A$ & $5,71 \mathrm{ab} B$ \\
\hline UFPI 689 Rajada & 19,33 & 4,17 & 39,3 a $A$ & 3,9 a $\mathrm{A}$ & $37,28 \mathrm{ab} A$ & $2,57 \mathrm{ab} A$ \\
\hline UFPI 689 Branca & 13,43 & 2,57 & 39,4 a $\mathrm{A}$ & 5,1 a $A$ & $43,28 \mathrm{ab} A$ & $4,0 \mathrm{ab} \mathrm{A}$ \\
\hline UFPI 701 & 14,43 & 3 & 39,3 a $\mathrm{A}$ & 4,1 a $\mathrm{A}$ & $39,0 \mathrm{ab} \mathrm{A}$ & $3,71 \mathrm{ab} A$ \\
\hline UFPI 720 & 22,8 & 2,6 & 43,9 a $A$ & 4,1 a $\mathrm{A}$ & $35,28 \mathrm{~b} \mathrm{~A}$ & $3,0 \mathrm{ab} \mathrm{A}$ \\
\hline UFPI 715 & 10,14 & 5 & 46,4 a $\mathrm{A}$ & 2,3 a A & $44,57 \mathrm{ab} A$ & $2,71 \mathrm{ab} A$ \\
\hline $\mathrm{F}$ & & & $0,72 \mathrm{~ns}$ & $1,35 \mathrm{~ns}$ & $2,19 * *$ & $2,58 * *$ \\
\hline $\mathrm{Cv} \%$ & & & 10,66 & 36,3 & 18,55 & 32,3 \\
\hline
\end{tabular}

ns Não significativo através do teste $\mathrm{F}(\mathrm{p}<0,05)$.

* Dados não analisados estatisticamente por não apresentar uniformidade.

** Médias seguidas de mesma letra minúscula na coluna não diferem estatisticamente pelo teste de Tukey $(\mathrm{p}<0,01)$.

Médias seguidas de mesma letra maiúscula nas linhas, para a mesma variável, não diferem estatisticamente pelo teste "t" de Student.

Comparando as médias de postura da geração parental com as das gerações seguintes, percebe-se que os insetos, que até então estavam sendo mantidos na criação estoque em outro acesso de feijão fava, quando colocados para ovipositarem nos acessos a serem testados, tiveram sua oviposição prejudicada pela mudança de substrato e ovipositaram menos. A média de número de ovos da geração parental variou de 6,57 a 28 ovos viáveis, respectivamente, nos genótipos UFPI 463 e UFPI 515 Rajada. Na geração seguinte variou de 45,7 e 40,1 ovos viáveis nos mesmos acessos, respectivamente. Isso revela o efeito da adaptação dos insetos aos acessos. Situação que pode ser mal interpretada, caso não aja metodologia adequada. Diante disso, sugere-se que nos testes de confinamento visando resistência aos carunchos sejam utilizados os dados da primeira geração em diante.

Girão Filho et al. (2012), observaram médias de número de ovos variando de 20,3 no acesso UFPI 582 a 38,8 no acesso UFPI 230 em teste de confinamento, avaliados logo 
em seguida à mudança de substrato de alimentação/oviposição.

Barbosa et al. (2000) trabalharam em quatro gerações com Z. subfasciatus e quatro acessos de feijão comum, dois resistentes e dois susceptíveis e observaram que tanto no acesso resistente Arcelinal quanto nos susceptíveis as médias de número de ovos viáveis aumentaram da $1^{\circ}$ para a $4^{\circ}$ geração, mostrando adaptação, no entanto, a resistência antibiótica não foi perdida.

Em relação à emergência de adultos e período médio de desenvolvimento de Z. subfasciatus, foram observadas diferenças significativas entre e dentro das gerações estudadas (Tabela 4).

Tabela 4. Médias de percentual de emergência e período médio de desenvolvimento de Zabrotes subfasciatus criados em feijão fava em duas gerações em condições controladas.

\begin{tabular}{|c|c|c|c|c|}
\hline \multirow{2}{*}{ Acessos } & \multicolumn{2}{|c|}{ Emergência \% } & \multicolumn{2}{|c|}{ PMD } \\
\hline & F1 & $\mathrm{F} 2$ & $\mathrm{~F} 1$ & $\mathrm{~F} 2$ \\
\hline UFPI 32 & 80,4 c A & 62,28 abc B & $32,46 \mathrm{cde}$ & 32,1 cde \\
\hline UFPI 34 & 72,75 abc $A$ & 71,45 abc $\mathrm{A}$ & 32,29 cde & 32,0 cde \\
\hline UFPI 121 & $63,5 \mathrm{abc} A$ & 67,10 abc $\mathrm{A}$ & 33,27 bcde & $32,9 \mathrm{bcd}$ \\
\hline UFPI 222 & 83,27 c A & 66,2 abc A & 32,44 cde & $33,1 \mathrm{bc}$ \\
\hline UFPI 243 & 64,52 abc $A$ & 76,42 abc B & $33,45 \mathrm{bcd}$ & $33,3 \mathrm{abc}$ \\
\hline UFPI 251 & 77,44 bc A & 79,9 abc A & 32,30 cde & $31,6 \mathrm{def}$ \\
\hline UFPI 281 & 67,97 abc A & 55,13 a $\mathrm{A}$ & $31,60 \mathrm{de}$ & 31,3 ef \\
\hline UFPI 463 & 76,86 bc A & 69,0 abc $\mathrm{A}$ & 32,0 cde & 32,4 cde \\
\hline UFPI 468 & $80,1 \mathrm{c} \mathrm{A}$ & 86,7 с A & $31,35 \mathrm{e}$ & $30,3 \mathrm{f}$ \\
\hline UFPI 486 & $78,85 \mathrm{c} \mathrm{A}$ & 68,68 abc A & $31,75 \mathrm{cde}$ & $31,5 \mathrm{def}$ \\
\hline UFPI 500 & 72,76 abc $A$ & 67,11 abc $A$ & 32,70 cde & 32,2 cde \\
\hline UFPI 504 & 68,45 abc $A$ & $57,32 \mathrm{ab} A$ & 31,49 de & $31,6 \mathrm{def}$ \\
\hline UFPI 515 & 71,87 abc A & 69,07 abc A & $33,72 \mathrm{bc}$ & 32,0 cde \\
\hline UFPI 579 & 49,28 a $\mathrm{A}$ & 60,64 abc B & $32,98 \mathrm{cde}$ & $34,1 \mathrm{ab}$ \\
\hline UFPI 581 & 77,45 bc A & 81,3 bc A & 32,88 cde & 32,5 cde \\
\hline UFPI 654 & 69,97 abc A & 74,14 abc $\mathrm{A}$ & 33,21 bcde & 32,8 bcde \\
\hline UFPI 666 & 71,37 abc $\mathrm{A}$ & 65,76 abc $A$ & $32,56 \mathrm{cde}$ & 32,3 cde \\
\hline UFPI 676 & 77,50 bc A & $60,91 \mathrm{abc} B$ & 32,71 cde & 32,5 cde \\
\hline UFPI 689 Rajada & $53,91 \mathrm{ab} \mathrm{A}$ & 63,35 abc $A$ & 31,94 cde & $33,3 \mathrm{abc}$ \\
\hline UFPI 689 Branca & 75,85 bc $\mathrm{A}$ & 64,9 abc A & $32,65 \mathrm{cde}$ & $33,5 \mathrm{abc}$ \\
\hline UFPI 701 & $65,41 \mathrm{abc} A$ & $58,42 \mathrm{ab} \mathrm{A}$ & $36,65 \mathrm{a}$ & $34,0 \mathrm{ab}$ \\
\hline UFPI 720 & 66,92 abc $A$ & 66,6 abc $\mathrm{A}$ & $35,26 \mathrm{ab}$ & $34,8 \mathrm{a}$ \\
\hline UFPI 715 & 72,70 abc $\mathrm{A}$ & 81,36 bc A & 33,17 cde & 32,3 cde \\
\hline $\mathrm{F}$ & $3,54 * *$ & $2,85 * *$ & $9,83 * *$ & $11,31 * *$ \\
\hline $\mathrm{Cv} \%$ & 24,16 & 24,42 & 3,06 & 2,36 \\
\hline
\end{tabular}

** Médias seguidas de mesma letra minúscula na coluna não diferem estatisticamente pelo teste de Tukey $(p<0,01)$.

Médias seguidas de mesma letra maiúscula nas linhas, para a mesma variável, não diferem estatisticamente pelo teste " $t$ " de Student.

Na geração F1, as médias de percentual de emergência de adultos variaram de 49,28 a 83,27\%, respectivamente, nos acessos UFPI 579 e UFPI 222. Estes mesmos acessos na geração seguinte se comportaram de maneira diferente. $\mathrm{O}$ acesso UFPI 579 aumentou a emergência de adultos para $60,64 \%$, demonstrando perda de resistência. Por outro lado o acesso UFPI 222 diminuiu a emergência para 66,2\% devido à sua antibiose. Na geração F2, as maiores diferenças de emergência de adultos foram observadas nos acessos UFPI 281 e UFPI 468 com 55,13 e 86,70 \% respectivamente, valores compatíveis com os da geração anterior, mas agora manifestados em outros acessos. Isso representa uma falta de estabilidade de resistência quando se leva em consideração as duas gerações, como ocorreu com o genótipo Arcelina1 no trabalho de Barbosa et al. (2000), o qual permaneceu com alta mortalidade de larvas de Z. subfasciatus por quatro gerações consecutivas. No entanto, embora com uma baixa mortalidade, pode-se observar que os acessos UFPI 32 e UFPI 676 diminuíram significativamente a emergência de adultos do caruncho $Z$. subfasciatus entre as gerações estudadas; UFPI 32 diminuiu 18,12\% e o UFPI 676 16,59\%. Por outro lado, aconteceu o oposto com os acessos UFPI 243 e UFPI 579 que aumentaram o percentual de adultos emergidos de uma geração para outra, respectivamente em 11,9 e 11,36\%. $\mathrm{O}$ restante dos acessos permaneceu estatisticamente constante nas duas gerações seguintes.

Girão Filho et al. (2012), trabalhando com oito acessos de feijão-fava, encontraram emergência de Z. subfasciatus variando de 79,21 no acesso UFPI 515 a 92,74 no acesso UFPI 494, valores que corroboram com os desta pesquisa e que são muito altos comparados aos que pode-se encontrar em P. vulgaris, 16,4 \% (BARBOSA et al., 1999).

Em relação ao PMD, pode-se observar que houve diferenças estatísticas entre as médias, que variaram de 31,35 a 36,65 dias na primeira geração e de 30,3 a 34,8 dias na segunda geração de Z. subfasciatus. Tanto na F1 quanto na F2, o acesso UFPI 468 apresentou as menores médias, diferindo, nos dois casos da mais alta (31,35 na F1 e 30,3 na F2), caracterizando uma alta suscetibilidade. Na geração F1, os acessos UFPI 701 e UFPI 720 apresentaram as maiores médias de PDM. Os acessos UFPI 689 rajada, UFPI 689 branca, UFPI 579, UFPI 701 e principalmente o UFPI 720 se destacaram com as maiores médias de PMD de $Z$. subfasciatus na geração F2. Dessa forma os acessos UFPI 701 e UFPI 720 se destacaram em ambas as gerações, apresentado resistência antibiótica neste parâmetro. Este parâmetro é 
muito importante na manifestação da resistência. Segundo Lara (1991) alimentos que proporcionam um aumento no ciclo biológico de um indivíduo são menos favoráveis ao desenvolvimento do mesmo e podem manter a população do inseto em níveis menores em relação a outro.

Girão Filho et al. (2012), trabalhando com acessos de feijão-fava, em relação à $Z$. subfasciatus, obtiveram médias de PMD variando entre 29,3 dias no acesso UFPI 468, que corrobora com os desta pesquisa, e 30,4 dias no acesso UFPI 482. No entanto, em feijão comum já foram observados acréscimos de até 17 dias no PMD de Z. subfasciatus, característica de alta antibiose (BARBOSA et al., 2000).

Tendo em vista que tanto o feijão-fava quanto o feijão do grupo carioca pertencem à ao mesmo gênero, e que, provavelmente tenham sido domesticadas nas mesmas regiões, norte da américa do sul e México (Lopes et al., 2010; Freitas, 2006) espera-se que dentro dos feijões-fava encontrese também alguma fonte de resistência assim como no feijão comum, visto que ambas são hospedeiras e coevoluiram junto com Z. subfasciatus.

\section{CONCLUSÕES}

O acesso UFPI 468 é altamente suscetível em relação à Z. subfasciatus.

O acesso UFPI 701 é o mais resistente dentre os acessos de Phaseolus lunatus testados em relação à Z. subfasciatus.

Os acessos UFPI 121, UFPI 222, UFPI 281, UFPI 504, UFPI 579, UFPI 689 Rajada, UFPI 701 e UFPI 720 apresentam moderada antibiose em relação à Z. subfasciatus.

\section{REFERÊNCIAS}

BALDIN, E. L. L.; PEREIRA, J. M. Resistência de genótipos de feijoeiro a Zabrotes subfasciatus (Bohemann, 1833) (Coleoptera: Bruchidae). Revista Ciência e Agrotecnologia, v.34, n.6, p.1507-1513, 2010.

BARBOSA, F. R., YOKOYAMA, M., PEREIRA, P. A. A., ZIMMERMANN, F. J. P. 1999. Efeito da proteína arcelina na biologia de Zabrotes subfasciatus (Boheman 1833) em feijoeiro. Pesquisa Agropecuária Brasileira, v.34, n.10, p.1805-1810, out. 1999.

BARBOSA, F. R.; YOKOYAMA, M.; PEREIRA, P. A. A.; ZIMMERMANN, F. J. P. Danos de Zabrotes subfasciatus (Boh.) (Coleoptera: Bruchidae) em Linhagens de Feijoeiro (Phaseolus vulgaris L.) Contendo Arcelina. Anais da Sociedade Entomológica do Brasil, v.29, n.1, p.113-121, 2000.

BLAIR, M. W.; PRIETO, S.; DIAZ, L. M.; BUENDIA, H. F.; CARDONA, C. Linkage disequilibrium at the APA insecticidal seed protein locus of common bean (Phaseolus vulgaris L.). BMC Plant Biology, 10 : 79-94. 2010.

BOTEGA, D. B.; RODRIGUES, C. A.; JESUS, F. G.; SILVA, A. G.; PEIXOTO, N. Resistência de Genótipos de Feijão-vagem ao Ataque de Bruquíneos, em Condições de Laboratório. Revista Caatinga, v.25, n.1, p.92-97, 2012.

COSTA, E. N.; SOUZA, B. H. S.; BOTTEGA, D. B.; OLIVEIRA, F. Q., RIBEIRO, Z. A.; BOIÇA JUNIOR, A. L. Divergência genética de genótipos de feijoeiro a infestação de Zabrotes subfasciatus (Bohemann) (Coleoptera: Bruchidae). Semina, v.34, n.6, p.2737-2752, 2013.
FRANÇA, S. M.; OLIVEIRA, J. V.; ESTEVES FILHO, A. B.; OLIVEIRA, C. M. Toxicity and repellency of essential

FREITAS, F. O. Evidências genético-arqueológicas sobre a origem do feijão comum no Brasil. Pesquisa agropecuária brasileira, Brasília, v.41, n.7, p.1199-1203, 2006.

GIRÃO FILHO, J. E.; ALCÂNTARA NETO, F., PÁDUA, L. E. M.; PESSOA, E. F. Repelência e atividade inseticida de pós vegetais sobre Zabrotes subfasciatus Boheman em feijãofava armazenado. Revista Brasileira de Plantas Medicinais, Campinas, v.16, n.3, p.499-504, 2014.

GIRÃO FILHO, J. E.; PÁDUA, L. E. M.; PESSOA, E. F; SILVA, P. R. R. Antibiose e antixenose de feijão-fava a Zabrotes subfasciatus (Boh.) (Coleoptera: Chrysomelidae: Bruchinae). Arquivos do Instituto Biológico, v.83, p. 1-6, 2016

GIRÃO FILHO, J. E.; PÁDUA, L. E. M.; SILVA, P. R. R.; GOMES, R. L. F.; PESSOA, E. F. Resistência genética de acessos de feijão-fava ao gorgulho Zabrotes subfasciatus (Boh.) (Coleoptera: Bruchidae). Comunicata Scientiae, v.3, n.2, p.84-89, 2012.

IBGE - Instituto Brasileiro de Geografia e Estatística. Produção Agrícola Municipal, 2010, 2011, 2012. Disponível em:http://www2.sidra.ibge.gov.br/bda/agric/default.asp?t=1\& $\mathrm{z}=\mathrm{t} \& \mathrm{o}=11 \& \mathrm{u} 1=1 \& \mathrm{u} 2=1 \& \mathrm{u} 3=1 \& \mathrm{u} 4=1 \& \mathrm{u} 5=1 \& \mathrm{u} 6=1<$ Acesso em 6 dez. 2013>

LARA, F. M. Princípios de resistência de plantas a insetos. 2. ed. São Paulo: Ícone, 1991. 336p.

LOPES, A. C. A.; GOMES, R. L. F.; SILVA, R. N. O.; COSTA, E. M. R.; SOUSA, I. F. S.; SANTOS, J. O.; SOUSA, T. H. P.; SILVA, K. J. D. Diversidade Genética. In: Araújo, A. S. F., Lopes, A. C. A., Gomes, R. L. F. A cultura do feijão-fava na Região Meio-Norte do Brasil. EDUFPI, Teresina, Brasil, 2010. 272p.

MIKAMI, Y. A.; PISSINATI, A.; FAGOTTI, D.; MENESES JÚNIOR, A. O.; VENTURA, M. U. Control of the Mexican bean weevil Zabrotes subfasciatus with kaolin. Ciência Rural v.40, n.7, p.1497-1501, 2010.

MORAES, C. P. B.; BOIÇA JÚNIOR, A. L.; SOUZA, J. R.; COSTA, J. T. Determinação dos tipos de resistência em genótipos de feijoeiro ao ataque de Zabrotes subfasciatus. Revista Ceres v.58, n.4, p.419-424, 2011.

oils to Zabrotes subfasciatus (Boheman) (Coleoptera, Chrysomelidae, Bruchinae) in Phaseolus vulgaris L. Acta Amazonica, [online] v.42, n3, p.381-386, 2012.

RIBEIRO-COSTA, C.S.; PEREIRA, P.R.V.S.; ZUKOVSKI, L. Desenvolvimento de Zabrotes subfasciatus (Boh.) (Coleoptera: Chrysomelidae, Bruchinae) em genótipos de Phaseolus vulgaris L. (Fabaceae) cultivados no Estado do Paraná e contendo arcelina. Neotropical Entomology, v.36, n.4, p.560-564, 2007.

ZAUGG, I.; MAGNI, C.; PANZERI, D.; DAMINATI, M. G.; BOLLINI, R.; BENREY, B.; BACHER, S.; SPARVOLI, F.. QUES, a new Phaseolus vulgaris genotype resistant to common bean weevils, contains the Arcelin- 8 allele coding for new lectin-related variants. Theoretical Applied Genetics, v.126, n.3, p.647-661, 2013. 